\title{
Qualitative Description on Characteristics of Gravitational Field
}

\author{
Duan Xianxiang
}

Abstract

The paper proposes that the gravitational field changes atomic clock time. In this paper, the gravitational field is analogous to the electromagnetic field and there is a "mass field", so the universal gravitation equation is modified. In this paper, we propose an aether model, modify Maxwell's equations and add the reference frame.

Keywords: Characteristics of gravitational field, mass filed, intensity of gravitational field, torsion, Doppler effect, viscosity

\section{Characteristics of gravitational field}

\subsection{Characteristics of gravitational field}

Through the analogy of gravitational field and electromagnetic field, several qualitative descriptions on characteristics of gravitational field can be put forward. First, there are two concepts of viscosity and viscous force. This paper shows that due to the effect of electricity, magnetic field force and gravitation, the oscillation frequency of particle in magnetic field, electrical filed or gravitational field can be decreased. This phenomenon of decrease is viscosity and the force which decreases this frequency is viscous force.

(0) The etheric around a celestial body refracts or bends light.

Etheric resemble gravitational fields or Earth's atmosphere, with Etheric around every planet.

( 1 ) The gravitation of gravitational field can distort the motion trajectory and velocity of object and particle, and even change the shape and physicochemical characteristics of object. The gravitational field can stick the particle or the oscillation frequency of wave, such as electromagnetic wave and various particles.

(2) The gravitational field and electromagnetic field both have intensity. The greater the mass of gravitation source object or star, the greater the intensity of gravitational field. The higher the intensity of gravitational field, the bigger the 
viscous force of oscillation frequency ofparticle.

(3 ) The gravitational field has a physical quantity of "density". Around the object with small volume and big mass, the density of gravitational field is great as well as the intensity of gravitational field. The higher the density of gravitational field, the bigger the viscous force of oscillation frequency ofparticle.

(4) The intensity and density of gravitational field are related to the distance of gravitation source. The closer the distance, the greater the intensity and density.

(5) The gravitational field has torsion.

This paper makes an analogy of gravitational field and electromagnetic field. The electromagnetic field has the electromagnetic wave. And the electromagnetic wave consists of the electrical filed and magnetic field. And then this paper thinks that the gravitational wave also consists of the gravitational field and another filed. This filed is called "mass filed".

This paper also thinks that the gravitation can have an analogy with Lorentz force and the formula of Lorentz force is:

$$
F=q E+q v B
$$

$\mathrm{F}$ is Lorentz force, $\mathrm{q}$ is quantity of electric charge of charged particle, $\mathrm{E}$ is the intensity of electrical filed, $\mathrm{V}$ is the velocity of charged particle and $\mathrm{B}$ is the magnetic induction density.

The formula ofNewtonian gravitation is:

$$
F=G \frac{M m}{r^{2}}
$$

The formula of electrical filed strength is:

$$
E-K \frac{Q}{r^{2}}
$$

In the formula of Lorentz force, the electrical filed force is:

$$
F=q E=K \frac{Q q}{r^{2}}
$$

The quantity of electric charge $q$ and $Q$ can be thought of as the mass $m$ and $M$. the Newtonian gravitation can be thought of as the electrical filed force. Therefore, if the gravitational wave consists of the gravitational field and mass filed, there is also a force which is similar to Lorentz force magnetic force in the gravitation, and this 
force is mass filed force, which is called "torsion" here.

Through the analogy of the gravitational field and the electromagnetic field, it is assumed that a planet moves in the gravitational field of its parent star, the mass of the planet is $\mathrm{m}$.

Therefore, the mass filed force (torsion) is:

$$
F=m v B
$$

$v$ is the velocity ofplanet and B is the intensity of mass filed ofparent star.

The gravitation should be thought of as the Lorentz force, but only the electrical filed force is thought of as in Newtonian gravitation. And the formula of Newtonian gravitation can be corrected as:

$F=G \frac{M m}{r^{2}}+m v B(1)$

$m$ is the mass ofplanet, $\quad v_{1}$ is the velocity ofplanet, $B_{2}$ is the intensity of mass field

ofparent star, $M$ is the mass ofparent star, $v_{2}$ is the velocity of movement ofparent

star relative to planet and $B_{1}$ is the intensity of mass field ofplanet.

Therefore, the parent star is related to the gravitation and velocity of movement of planet which moves in its gravitational field. Although it is not moved with high velocity, the formula of Newtonian gravitation also needs to be corrected. This formula is (1). If the velocities of relative movements of parent star and planet are high and the Doppler effect is caused, the formula (4) and the formula (1) can be combined.

Because this paper compares the gravitational field with the electromagnetic field, the equation of gravitation with the Coulomb force and the Lorentz force, and thinks that there is also a mass field. But the question is, can you make such a simple analogy? Does gravity and electromagnetic force really match one to one? The electromagnetic field is made up of "magnetic field" and "electric field", and gravity is also composed of "gravitational field" and "mass field"? Is there such a simple correspondence? In order to solve the problem of whether this analogy can be solved, a hypothesis is proposed for verification. 
Gravitational waves have two fields, one mass field and one gravitational field.

Since the gravitational field has torsion, the particle moving in the gravitational field are the same as the particle moving in the magnetic field that their oscillation frequency will change because of velocity of movement. The higher the velocity of the movement, the bigger the torsion, and the lower the oscillation frequency of particle.

(6) Gravitational and Ether

This paper proposes an "etheric model".etheric is similar to the atmosphere or gravitational field, and there are etheric around objects, around the sun and around the earth. The etheric is wrapped in the earth or the sun and is relatively stationary with the earth or the sun. This paper argues that the gravitational pull of celestial bodies attracts special substances, which are "etheric", or the gravitational field is "other".

The ether can be likened to a "gravitational field," where planets radiate it. It has no boundary, but it gets weaker as it gets farther away. In the empty Spaces of the universe, the aether of the planets is mixed, but Surrounding the earth is primarily the etheric of the earth.

Ether can refraction light, close to the space of the celestial body, etheric density is large, far away from the space of the celestial body, etheric density is small, therefore, the space around the celestial body is like a convex lens, refracting light.

The sun rotates, its gravitational field will drive the ether spin together. The Earth rotates, and the Earth's gravitational field also drives the ether to spin together. As the Earth's surface ether revolves with the earth, the surface ether and the Earth remain relatively static. Thus, the interference fringes of the MM experimental interferometer at rest on the ground will of course not change. However, Miller's MM experiments on high mountains show that interference fringes change on high mountains, indicating that the ether on high mountains revolves with the sun, not with the earth.

You can also do experiments with airplanes, there are two planes, each with Michelson and Morey interferometers, flying east over the equator (the same direction 
as the Earth's rotation) and one west. If the interference fringes move.In the MM experiment on an airplane, the interferometer also changes, but like Mille's alpine experiment, the interferometer moves relative to the ether of the sun.

If this model is correct, the interferometer of the Michelson experiment at rest on the ground will not have interference fringes, because the ether around the earth revolves with the earth, and the ether is stationary relative to the earth.

But if so this etheric model is applicable.

1.2 Judgement of the direction of each vector in gravitational field and mass field

In common with the electromagnetic field, there should be similar right hand grip rule and left-hand rule in the directions of each vector in gravitational field and mass field. It takes the gravitational field analogous to electric field and mass field to magnetic field. In electromagnetic field, electric field and magnetic field are mutually perpendicular. Therefore, take the gravitational field analogous to that, and gravitational field and mass field are mutually perpendicular too.

The directions of each vector in mass field are determined by left-hand rule. The "current direction" is analogous to the direction of motion v of planet and "magnetic induction line" is analogous to the direction of the mass field ofparent star. Therefore, the direction of thump is the direction of mass field force (torsion).

\section{Explanation on experiment to prove relativity}

2. 1 The starlight deflects around the celestial body, and gravitational lens.

This is mainly because the etheric around the celestial body refracts or bends light.

2.2 Gravitational redshift of light ray of white dwarf. Since the gravitational field of white dwarf is strong, the gravitational field sticks the oscillation of light wave and decreases the frequency of light wave, which lengths the wavelength of light wave.

2.3 The atomic clock of American Bureau of Standard, which is located at the altitude of $1650 \mathrm{~m}$, is 5 microseconds faster than that of Royal Greenwich Observatory, which is located at the altitude of $25 \mathrm{~m}$. The reason is that the place with low altitude is closer to mass point of earth and its oscillation frequency of atom is lower than that of 
the place with high altitude. Therefore, the oscillation frequency of atom of atomic clock of American Bureau of Standard is higher and faster than that of Royal Greenwich Observatory.

\subsection{Reason for GPS clock becomes fast.}

Two gravitational fields and characteristics have been played a role in this phenomenon. One is that the intensity of gravitational field is related to the distance of gravitation source, which is the characteristic of gravitation in above (4). The distance of satellite to the gravitation source of earth is further than the distance of clock on the ground to earth. The gravitation of the location of satellite is much less and the oscillation frequency of atom of atomic clock on the satellite is higher. In addition, the movement of satellite generates the torsion through the characteristic of gravitation in (5). But because it is the circle movement with low velocity, the effect of torsion is little. Therefore, the characteristic of gravitation in (4) plays a role in general and the atomic clock of satellite is faster than that in the ground.

2.5 Lifetime extension of $\mu$ in high-speed movement. When it moves at high speed in gravitational field or magnetic field, its oscillation frequency is decreased due to the viscous effect of mass field torsion or magnetic force on its oscillation frequency. It saves the energy which is used for oscillation to relatively increase the energy of decay, and the decay period lengthens.

2.6 In 1970, Hafir and Keating completed an experiment named "Circumnavigation experiment of carrying atomic clock by plane"

The experiment content is: put four cesium atomic clocks on two planes separately, one plane flies eastward and the other one flies westward. And two planes fly around the equatorial plane with high speed. As a result, the atomic clocks on the plane eastward become slow and the atomic clocks on the plane westward become fast.This is because the plane flies in the etheric of the sun. An atomic clock flying eastward and an atomic clock flying westward are interacting with the sun's etheric. In line with the direction of rotation, that is, flying eastward, the atomic clock is subjected to the resistance of the ether, the frequency of the cesium atom oscillates is lower, and the time is slower. Flying westward, counteracting the Earth's rotation, 
cesium atoms oscillate with high frequency and fast time. Because of the interaction of gravitational field and etheric field, the oscillation frequency of cesium atoms on the ground is faster than that of "flying eastward" and slower than that of "flying westward".

2.7 Gravitational Lensing. Gravitational Lensing is the characteristics (5) of gravitational field in preamble, which is the effect of torsion. Einstein ring is formed by twisting the light by torsion.

All in all, gravitational field can change the motion trajectory of the object in field; and it can change the oscillation frequency of particle. The oscillation frequency of atomic clock is changed, but the time is unchanged. The gravitational field change the time of a clock is essentially the same with that human adjusts a watch. Human

adjusts the time, which does not change the time, namely: clock $\neq$ time

This is because, time is not matter, it is not substance. "time" does not exist. It is simply a "ruler" used by man to indicate the movement of objects, or the speed of material change. It is an abstract concept, and like the Arabic numeral $1 / 2 / 3$, in nature, the universe does not really exist, so time is immutable.

\section{NATURE OF TIME AND THE NATURE OF SPACE}

Time doesn't exist. Time is an imaginary cognitive tool for human beings to compare the speed of things moving and changing. There is no "time" in the universe, but an abstract concept created by human beings. But it's so old, and it wasn't founded by a specific scientist, that many people forget where it came from: that humans happen to be all fiction.

Human beings make up and define time, and "simultaneity" is the meaning of its existence. If time does not have simultaneity, it cannot be "faster or slower", And time has no meaning. So time is the same everywhere in the universe and cannot be changed.

Similarly, space does not exist. Space is also an imaginary abstraction, it is also defined, the whole universe can only define one kind of space, namely the space of Euclidean geometry. A straight line is a straight line, and you can't define a curve as a straight line, which would be confusing.

Therefore, we can either define the universe as Euclidean or non-Euclidean, and we can't think of the universe as both Euclidean and non-Euclidean, because there is identity in space, the whole universe is the same.

Since space is also a cognitive tool, it does not exist and cannot be "distorted". To distort is to tamper with its definition.

\section{Modification of maxwel I's equations}


4. 1 Abe-maxwel I's law

The original formula is:

$\int_{l} B \cdot d l=\mu_{0}\left(I_{e n c}+\varepsilon_{0} \frac{d}{d t} \int_{S} E \cdot d a\right)$

After modification:

$\int B \cdot d l=\mu_{0} \frac{v \pm v_{y}}{v \mp v_{d}}\left(I_{e n c}+\varepsilon_{0} \frac{d}{d t} \int_{s} E \cdot d a\right)$

$\frac{v \pm v_{y}}{v \mp v_{d}}$, Which affects the rate of change of electric flux. $\frac{d}{d t} \int_{s} E \cdot d a$

$d_{t}^{d} \int_{s} E \cdot d a$ is the rate of Electrical flux change when the curved surfaces and electric field source are stationary in the medium.

When the curved surfaces move in the medium, the change rate of the curved surfaces Electrical flux is different from that at rest, so add a $\frac{v \pm v_{y}}{v \mp v_{d}}$.

Same as the Doppler effect, $v$ Is the velocity of the electric field, $v_{y}$ is the velocity of the curved surfaces (curved surfaces of the electric flux needs to be calculated) relative to the medium(moving towards the electric field source), if close to the emission source, the forward is "+", away from the “-'. $v_{d}$ is the velocity of the emission source(electric field) in the medium, if close to the curved surfaces, the forward is "-", away from the "+".

This is done because, if the electromagnetic wave requires a medium, the rate of change of electric and magnetic flux as the electric and magnetic field sources move through the medium is affected by the speed of their electric field sources (magnetic field sources, curved surfaces).

Analogous to the doppler effect formula, the change rate and frequency of electric flux are similarities. $f=\left(\underline{\underline{v} \pm} \underline{v_{0}}\right) f$ $v \mp v_{s}$

In the doppler effect, the frequencyf received by the observer is affected by the velocity 
$v$ of the wave in the medium and by the velocity $v_{0}$ of the observer and the velocity $v_{s}$ of the source. Similarly, the change speed of the electric flux of the curved surfaces is also affected by the motion speed of the curved surfaces, motion velocity of the emission source and the propagation speed of the electric field.

By analogy with doppler effect, the curved surfaces is regarded as the observer. The relationship between the electric flux change rate of the curved surfaces and the electric flux change rate of the emission source is as follows:

$$
d t \int_{y} E \cdot d a=\frac{v \pm v_{y}}{v \mp v_{d}} \frac{d}{d t} \int_{s} E \cdot d a
$$

Where, the left side is the rate of electric flux change of the curved surfaces. ${ }_{d t}^{t} \int_{s} E \cdot d a$ is the flux of the curved surfaces when the curved surfaces and emission source are stationary in the medium. $v_{y}$ is the velocity of the curved surfaces (curved surfaces of the electric flux needs to be calculated) relative to the medium. and $v_{d}$ is the velocity of the emission source in the medium.

Similarly, the rate of change of magnetic flux can be modified in this way. Maxwell's third equation can be modified as follows:

$$
\int_{c} E \cdot d l=-\frac{v \pm v_{\mathrm{e}}}{v \mp \underline{v}_{s}} \int_{\partial t}^{\underline{\partial} \underline{B}} \cdot d a
$$

$v$ It's the velocity of the magnetic field, $v_{c}$ it's the velocity of the curved surface in the medium.

$v_{z}$ is the velocity of the emission source(magnetic field).

The law of gauss electric field and the law of gauss magnetic field in maxwell's equations need not be modified, but it should be noted that they are only formulas in the static state.

\section{Gravitational field equation comjecture}

$$
\oint Y \cdot n d a=\frac{m}{x_{0}}
$$

$Y$ is the strength of the gravitational field and $x_{0}$ is the constant of the gravitational field (similar to the dielectric constant).

The formula says that a body generates a gravitational field, and the flux of the gravitational field through any closed surface is proportional to the total mass that the surface contains.

$$
\oint_{s} B \cdot n d a=0 \text { (2) }
$$

$B$ is the strength of the mass field (similar to the magnetic field strength in Maxwell's equations). The formula says that the flux of a mass through any closed surface is zero.

$\oint_{c} Y \cdot d l=-\frac{v \pm v_{y}}{v \mp v_{d}} \frac{d}{d t} \int_{s} B \cdot n d a$

The formula means:

A gravitational field is induced by a change in flux across a surface mass field. 
$\oint_{c} B \cdot d l=Z_{0} \frac{v \pm v_{y}}{v \mp v_{d}}\left(\frac{m}{t}+x_{0} \frac{d}{d t} \int_{s} Y \cdot n d a\right)$

$Z_{0}$ It's the mass field propagation efficiency, analogous to permeability

The formula means:

The flux across $\frac{m}{t}$ a surface or changing gravitational field produces a mass field.

The reason for this is that the analogy between gravitational field and electromagnetic field, that "mass" is "electromagnetic field," "gravitational field" is "electric field," and "mass field" is

"magnetic field."

\section{Wave-particle duality is explained by med ium}

You can compare light waves to sound waves.

When the sound is turned on, the diaphragm vibrates, exerting a force on the air close to it, or rather on the air molecules. When a molecule moves under a force, because there are so many molecules, Air molecules close to the vibrating membrane (assuming they form a "plane" or "wall") collide with the outer molecules, which, on the one hand, move perpendicular to the direction of the wave, forming a crest. On the 
other hand, they deliver collisions to molecules further out. Since the vibration of the trumpet membrane is intermittent, so are the molecular collisions. And that forms the wavelength.

The molecules in the inner layer collide with the molecules in the outer layer, and the outer molecules collide with the outer layer, transmitting this "collision" forward, which forms the sound wave.

Sound waves are the result of collisions between air molecules,air molecules have another form of motion: wind. Air molecules move to form "wind".

When air molecules move in the form of "wind", they are actually in the form of "particles", and when they move in the form of "acoustic collide", they are "waves". Therefore, the movement of air molecules is also wave-particle duality.

Compare light waves with sound waves. The flashlight gives off light, like sound machine speakers sound. The dielectric of light also made up of particles that resemble air molecules. The light wave dielectric (also made up of particles similar to air molecules) near the flashlight collides with the outer dielectric particle. The outer dielectric particle transmits this collision. And the dielectric particle is collided into photons, which form the light visible to the naked eye.

Thus, the wave phase of light is formed by the collision of dielectric particles of light and the transfer of "collisions".while the motion in the form of particles is the 'peaceful' motion of the medium particles as a whole, just like the 'wind'. At this time, there is no violent collision between the medium particles.

The flashlight does not emit photons, it only provides energy for the light medium particles to collide with each other, which are excited and become bright, forming the light column.

Thus, the wave phase of light is formed by the collision of the dielectric particles of light, which act as particles when they move like the "wind" of air molecules. In this way, it can be seen that the wave-particle duality of light is actually the duality of the dielectric particles of light. Essentially, "wave-particle duality" is just two different modes of motion for dielectric particles.

Light is just the motion ofparticles of light medium. Photons do not exist, just as 
phonons do not exist in sound waves. If the existence ofphotons is insisted on, then "photons" are light medium particles and "phonons" are air molecules.

Therefore, if there is a medium in the light wave, the wave-particle duality can be reasonably explained. If there are no "dielectric particles" and only photons, it is difficult to imagine that the photons behave as both waves and particles.

\section{Reference}

[1] Wang Shaojie, Gu Mu, Wang Zuyuan. College Physics [M]. Beijing: Higher Education Press, 2017. 